\title{
Caracterización sobre la toma de decisiones éticas en las unidades de cuidado neonatal por parte de pediatras y neonatólogos
}

\author{
Characterization of making ethical decisions in neonatal care \\ units by pediatricians and neonatologists
}

\section{Caracterização em relação à deliberação ética nas unidades de cuidados neonatais por pediatras e neonatologistas}

\author{
Sergio Iván Agudelo-Pérez, MD., Esp. * \\ María José Maldonado-Calderón, MD., Esp. ** \\ Carlos Pinzón-Flórez, MD., Mg. *** \\ Carlos Pérez-Barreto $* * * *$ \\ María Mazzanti-di-Ruggiero, B., Esp, PhD. *****
}

\section{Resumen}

Introducción: Ha aumentado la sobrevida de los neonatos críticamente enfermos, lo que ha llevado a los profesionales encargados del cuidado de estos bebés a enfrentar frecuentemente decisiones éticas. En el presente trabajo se plantea el objetivo de caracterizar las situaciones éticas que enfrentan, el criterio que utilizan y la forma en que se toman las decisiones en los dilemas éticos por parte de los pediatras y neonatólogos. Métodos: Se realizó un estudio de corte transversal. Se invitaron a participar 87 neonatólogos y/o pediatras que quisieran contestar de forma voluntaria la encuesta para la caracterización y trabajaran en unidades neonatales de Chía y Bogotá entre el 1 de octubre de 2014 y 31 de enero de 2015 , de los cuales aceptaron participar 45 profesionales $(51.7 \%)$. Se realizó un análisis exploratorio de los datos, utilizando estadística descriptiva. Resultados: De los profesionales que contestaron la encuesta, el $100.0 \%$ se ha enfrentado a problemas éticos, el $60.0 \%$ han recibido algún tipo de capacitación en bioética, $33.0 \%$ se apoya en comités de ética, $98.0 \%$ tienen algún límite de viabilidad para iniciar reanimar y el $93.0 \%$ ha limitado el esfuerzo terapéutico; el $98.0 \%$ incluye a los padres en las decisiones y registra la decisión en la historia clínica. Conclusiones: Son frecuentes los conflictos éticos en la unidad neonatal. La mayoría cuenta con capacitación y comité de ética para la toma de decisiones. Entre los especialistas hay opiniones heterogéneas sobre ciertos problemas éticos en las unidades neonatales. [Agudelo-Pérez SI, Maldonado-Calderón MJ, Pinzón-Flórez C, Pérez-Barreto C, Mazzanti-di-Ruggiero M. Caracterización sobre la toma de decisiones éticas en las unidades de cuidado neonatal por parte de pediatras y neonatólogos. MedUNAB 2016; 19(1): 9-17]

Palabras clave: Recién Nacido; Ética Clínica; Cuidado Intensivo Neonatal; Cuidado Terminal; Neonatología.

\footnotetext{
Médico especialista en Pediatría, Perinatología y Neonatología, Profesor asistente, Facultad de Medicina, Universidad de La Sabana y Clínica de la universidad de La Sabana, Colombia.

** Médico especialista en Pediatría, Profesor de Pediatría, Facultad de Medicina, Universidad de La Sabana, Colombia.

*** Médico general, Magister en Epidemiología, Profesor asistente departamento de Investigación, Facultad de Medicina, Universidad de La Sabana, Colombia. **** Estudiante, Facultad de Medicina, Universidad de La Sabana, Colombia.

***** Bióloga, Especialista en Bioética, Doctor en Bioética, Directora departamento de Bioética, Facultad de Medicina, Universidad de La Sabana, Colombia
}

Autor de correspondencia: Sergio Iván Agudelo Pérez, Profesor asistente, Facultad de Medicina, Universidad de La Sabana, Edificio H. Oficina 204D, Campus del puente del Común Km 7 Autopista Norte de Bogotá, Chía, Cundinamarca, Colombia. Correo electrónico: sergioap@clinicaunisabana.edu.co. 


\section{Abstract}

The survival of critically ill neonates has increased, which has led to professional caregivers of these babies to face ethical decisions about it. This paper outlines the aim of characterizing ethical situations that caregivers face, the criterion they use and how decisions on ethical dilemmas are taken by Pediatricians and Neonatologists. Methodology: A cross-sectional study was performed. 87 neonatologists and/or Pediatricians, who work in neonatal care units in Chia and Bogota between October 1 of 2014 and January 31 of 2015, were invited to participate and answer a characterization survey voluntarily, but only 45 professionals $(51.7 \%)$ agreed to take part in it. An exploratory data analysis was performed by using descriptive statistics. Results: Professionals who answered the survey, $100.0 \%$ of them has faced ethical problems, $60.0 \%$ of them has received some training in bioethics, $33.0 \%$ relies on ethics committee, $98.0 \%$ has a viability limit to start reanimating and $93.0 \%$ has limited the therapeutic effort; $98.0 \%$ includes parents in decisions and registers the decision in the medical record. Conclusions: Ethical conflicts in the neonatal care unit are frequent. Most professionals have training and an ethics committee for decision-making. Among the specialists there are heterogeneous views on certain ethical problems in neonatal care units. [Agudelo-Pérez SI, MaldonadoCalderón MJ, Pinzón-Flórez C, Pérez-Barreto C, Mazzantidi-Ruggiero $M$. Characterization of making ethical decisions in neonatal care units by pediatricians and neonatologists. MedUNAB 2016; 19(1): 9-17]

Key words: Newborn; Infant; Clinical Ethics; Neonatal Intensive Care; Terminal Care; Physician's Practice Patterns; Neonatology.

\section{Introducción}

Los avances tecnológicos, de seguridad y calidad de la atención neonatal, como es el cuidado respiratorio, nutricional y antibióticos, entre otros, ha tenido como consecuencia el aumento de la sobrevida de los neonatos críticamente enfermos y en los límites de la viabilidad (1-4). Se ha reportado en la literatura que el $96.8 \%$ de profesionales en cuidado intensivo pediátrico y neonatal se enfrentan a decisiones de problemas éticos (5), principalmente relacionados con la limitación del esfuerzo terapéutico, el manejo de pacientes en los límites de viabilidad y con patologías que tienen implícitos pronósticos a corto y largo plazo muy sombríos. Por lo general, enfrentan la toma de estas decisiones en un contexto de incertidumbre (1, 6-9), generando estrés intelectual y emocional en los profesionales con la responsabilidad de tomarlas (1).

La disponibilidad de recursos tecnológicos puede llevar a su uso indiscriminado en neonatos críticamente enfermos, sin esperanza de recuperarse o con posibilidades de secuelas graves, con una intromisión en el proceso natural de la muerte, agonías prolongadas, sufrimiento del paciente y de sus familiares, elevando el costo de la atención $(7,10,11)$. La toma de la decisión se podría basar en dos visiones: una,

\section{Resumo}

Introdução: o aumento da sobrevivência de recém-nascidos criticamente doentes, o que levou a cuidadores profissionais para esses bebês muitas vezes enfrentam decisões éticas. Objetivo: Neste trabalho é caracterizar as situações éticas e os critérios utilizados por pediatras e neonatologistas no jeito como tomam as decisões, frente aos dilemas éticos surgidos. Métodos: O estudo realizado foi de tipo transversal. De 87 neonatologistas e / ou pediatras convidados a participar no estudo realizado entre 01 de outubro de 2014 e 31 de janeiro de 2015,45 profissionais $(51,7 \%)$, que trabalham nas unidades neonatais de Chia e Bogotá (Colômbia), aceitaram livremente responder as perguntas. Os dados foram estudados por meio de estatística descritiva. Resultados: As respostas da pesquisa mostraram que $100,0 \%$ dos professionais tem enfrentado problemas éticos, $60,0 \%$ receberam alguma preparação em bioética, $33,0 \%$ se apoia na comissão de ética, $98,0 \%$ têm um limite de viabilidade para começar a reanimação e 93,0\% tem limitado o esforço terapêutico; $98,0 \%$ inclui os pais nas decisões e o registra no prontuário. Conclusões: Os conflitos éticos são frequentes na unidade neonatal. A maioria tem formação e conta com a comissão de ética para tomar as decisões. Entre os especialistas há pontos de vista heterogêneos sobre certos problemas éticos nas unidades neonatais. [Agudelo-Pérez SI, Maldonado-Calderón MJ, Pinzón-Flórez C, PérezBarreto C, Mazzanti-di-Ruggiero M. Caracterização em relação à deliberação ética nas unidades de cuidados neonatais por pediatras e neonatologistas. MedUNAB 2016; 19(1): 9-17]

Palavras-chave: Recém-Nascido; Ética Clínica; Terapia Intensiva Neonatal; Assistência Terminal; Neonatologia.

estadística, en la que se tienen en cuenta las cifras de morbilidad y mortalidad de la unidad neonatal y el resultado y pronósticos a largo plazo de sus neonatos atendidos para tomar la decisión; y una visión o criterio individualizado, considerando el análisis médico y la voluntad de los padres (12). Esta decisión se relaciona con los principios éticos de no maleficencia, obligación de benefícencia y de justicia por el uso eficiente de recursos disponibles $(10,13)$.

En Colombia existe un porcentaje importante de nacimientos prematuros, de bajo peso al nacer y de bebés con patologías complejas, al tiempo que ha aumentado la oferta de unidades neonatales con mayor disponibilidad de camas para la hospitalización de bebés críticamente enfermos en unidades de alta complejidad. Actualmente se hace importante la formación y el conocimiento por parte del especialista de la unidad neonatal en temas de ética y de toma de decisiones difíciles, quién tiene que tomarlas y cómo debería ser el proceso de la decisión (14). En la literatura se reporta una variación en la forma en que los profesionales se enfrentan al mismo problema ético $(15,16)$. El conocer y caracterizar cómo se enfrentan los profesionales en nuestras unidades a estos problemas, podría ser el paso inicial para la generación de guías y recomendaciones en estos aspectos. En nuestro medio no encontramos datos comunicados entre 
profesionales de nuestro país sobre la frecuencia con que se toman estas decisiones, cómo se enfrentan a ellas y qué criterios utilizan para resolverlas. Nos planteamos como objetivo en este estudio, caracterizar la forma en que se toman las decisiones en los dilemas éticos por parte de los pediatras y neonatólogos en las unidades de cuidado neonatal de Sabana Centro y Bogotá, a qué situaciones éticas se enfrentan, el criterio que utilizan en la decisión, como es el proceso y registro al tomarlas, quienes se involucran en la decisión.

\section{Metodología}

Se realizó un estudio de corte transversal con un muestreo a conveniencia. Se invitó a participar a pediatras y/o neonatólogos que estuvieran trabajando en unidades neonatales de clínicas de Bogotá y Chía entre el 1 de octubre de 2014 y el 31 de enero de 2015, e ingreso voluntario para realizar la encuesta. Para la recolección y caracterización de la información, se diseñó una encuesta (Tabla 1) la cual se

Tabla 1. Encuesta.

\begin{tabular}{|c|c|c|}
\hline $\begin{array}{l}\text { Nivel de Complejidad de atención en salud de } \\
\text { la Unidad de Recién Nacidos en que labora: } \\
\text { - Nivel II de complejidad } \\
\text { - Nivel III de complejidad } \\
\text { - } \quad \text { Nivel IV de complejidad } \\
\text { ¿Durante su práctica clínica en la Unidad } \\
\text { Neonatal, se ha enfrentado a la toma de } \\
\text { decisiones éticas difíciles? } \\
\text { - Nunca } \\
\text { - } \quad 1 \text { vez } \\
\text { - Más de una vez } \\
\text { ¿Dispone el hospital/clínica/centro asistencial } \\
\text { de un comité de ética clínica para apoyo en } \\
\text { decisiones éticas? } \\
\text { - Si } \\
\text { - No } \\
\text { ¿En su Unidad de Recién Nacidos hay } \\
\text { guías o políticas de cuidado paliativo o de } \\
\text { acompañamiento al final de la vida? } \\
\text { - Si } \\
\text { - No } \\
\text { ¿Si usted tiene alguna convicción religiosa } \\
\text { esta influye en la toma de decisiones éticas? } \\
\text { Si }\end{array}$ & 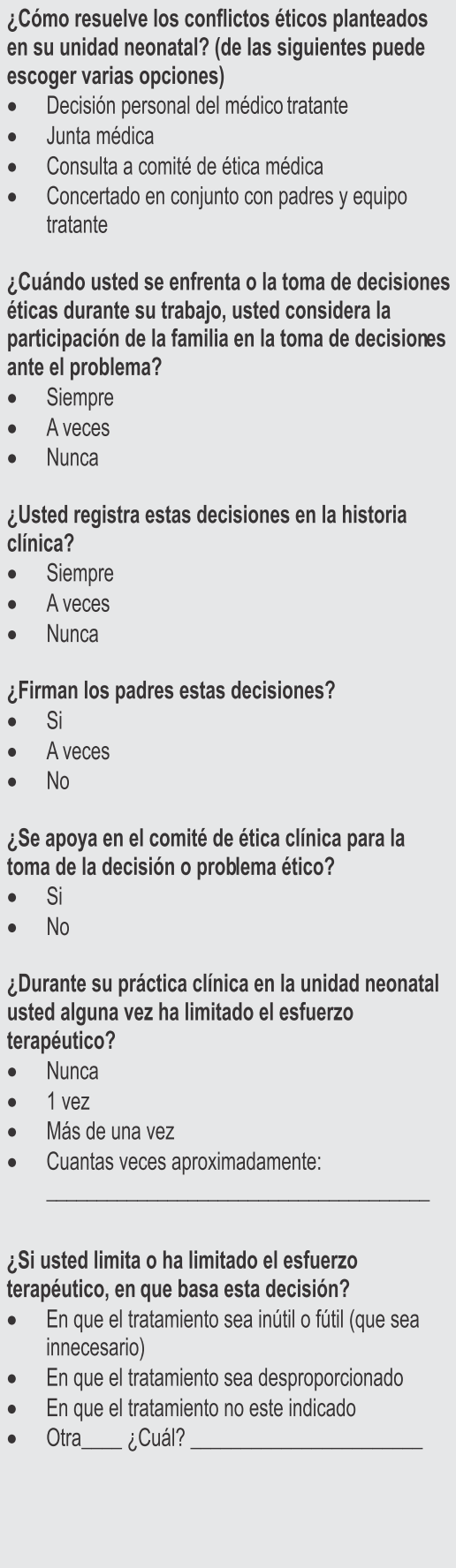 & $\begin{array}{l}\text { Usted considera dentro de su práctica clínica en la unidad } \\
\text { neonatal limitación del esfuerzo terapéutico como: (de las } \\
\text { siguientes puede escoger varias opciones) } \\
\text { - No iniciar reanimación } \\
\text { - } \quad \text { Retirar soporte ventilatorio invasivo o no invasivo } \\
\text { - No iniciar soporte ventilatorio invasivo o no invasivo } \\
\text { - } \quad \text { Retirar soporte vaso activo } \\
\text { - No iniciar soporte vaso activo } \\
\text { - Retirar manejo antibiótico } \\
\text { - No iniciar manejo antibiótico } \\
\text { - Retirar nutrición parenteral } \\
\text { - No iniciar nutrición parenteral } \\
\text { - Retirar nutrición enteral } \\
\text { - No iniciar nutrición enteral } \\
\text { - Limita ingreso a la unidad neonatal } \\
\text { - No administrar líquidos endovenosos } \\
\text { - Otra_Cuál? } \\
\text { ¿Tiene usted algún límite de viabilidad (peso ylo edad } \\
\text { gestacional) establecido para iniciar reanimación en sala de } \\
\text { adaptación? } \\
\text { - Si } \\
\text { - No }\end{array}$ \\
\hline
\end{tabular}

Fuente: Elaboración propia de los autores. 
aplicó de forma presencial. Se plantearon 24 preguntas agrupadas en datos básicos de los encuestados y unidades donde trabajan, el procedimiento para la resolución del problema ético y su opinión ante diferentes problemas éticos. Para las preguntas que evaluaban las preferencias se aplicó una escala Likert de 5 opciones de respuesta. Previo al inicio del estudio se aplicó una encuesta piloto a 5 pediatras y/o neonatólogos de una unidad neonatal de III nivel en Bogotá; con posterioridad a esta prueba se continuó con la encuesta original. La información fue tabulada empleando el programa Microsoft Excel 2013 licenciado para Windows 8 y procesada en el programa STATA. Se realizó un análisis exploratorio de los datos, utilizando estadística descriptiva, usando medidas de tendencia central, ubicación y dispersión para las variables continuas y frecuencias absolutas y relativas para las variables categóricas para la presentación y análisis de la información.

\section{Resultados}

Se invitaron a participar 87 pediatras y/o neonatólogos de 20 unidades neonatales, de los cuales aceptaron participar y contestaron de forma completa la encuesta 45 profesionales $(51.7 \%)$. De los 45 profesionales que contestaron la encuesta, $60.0 \%$ eran neonatólogos, $89.0 \%$ trabajan en unidades neonatales de alta complejidad, $86.7 \%$ tienen más de 5 años de experiencia laboral, el 100.0\% se ha enfrentado a problemas éticos (Tabla 1).

El 60.0\% (n27) utilizan más de una forma de resolver el problema ético; $78.0 \%$ lo concierta con los padres y el equipo tratante. E1 98.0\% consideran la participación de la familia en la resolución del problema. El 76.0\% siempre registra la decisión en la historia clínica y el $64.0 \%$ con la firma de los padres (Tabla 2).

Tabla 2. Datos básicos de los profesionales y de la unidad donde laboran

\begin{tabular}{|c|c|}
\hline VARIABLE & n (\%)/Promedio (DS) \\
\hline Edad & 44.4 años (+/- 7.6) \\
\hline \multicolumn{2}{|l|}{ Sexo } \\
\hline Masculino & $23.0(51.0 \%)$ \\
\hline Femenino & $21.0(49.0 \%)$ \\
\hline \multicolumn{2}{|l|}{ Tipo de especialidad } \\
\hline Pediatra & $18.0(40.0 \%)$ \\
\hline Neonatólogos & $27.0(60.0 \%)$ \\
\hline Años de experiencia en unidad neonatal & 12.7 (DS +/-7.2) \\
\hline$<5$ años & $6.0(13.3 \%)$ \\
\hline 5 a 10 años & $14.0(31.2 \%)$ \\
\hline 11 a 20 años & $21.0(46.7 \%)$ \\
\hline 21 a 30 años & $4.0(8.8 \%)$ \\
\hline \multicolumn{2}{|c|}{ Capacitación previa en resolución de problemas éticos } \\
\hline $\mathrm{Si}$ & $27.0(60.0 \%)$ \\
\hline No & $18.0(40.0 \%)$ \\
\hline \multicolumn{2}{|l|}{ Nivel de complejidad unidad neonatal } \\
\hline Nivel II & $4.0(9.0 \%)$ \\
\hline Nivel III & $12.0(27.0 \%)$ \\
\hline Nivel IV & $28.0(62.0 \%)$ \\
\hline \multicolumn{2}{|c|}{ Se ha enfrentado a problemas éticos en su práctica clínica } \\
\hline $\mathrm{Si}$ & $45.0(100.0 \%)$ \\
\hline No & $0.0(0.0 \%)$ \\
\hline \multicolumn{2}{|c|}{ Frecuencia con la que se enfrentan a dilemas éticos } \\
\hline $1 \mathrm{vez}$ & $2.0(4.0 \%)$ \\
\hline $2-5$ veces & $7.0(16.0 \%)$ \\
\hline $6-10$ veces & $20.0(44.0 \%)$ \\
\hline $11-15$ veces & $4.0(9.0 \%)$ \\
\hline $16-20$ veces & $1.0(2.0 \%)$ \\
\hline $21-30$ veces & $2.0(4.0 \%)$ \\
\hline Más de 30 veces & $7.0(16.0 \%)$ \\
\hline
\end{tabular}




\begin{tabular}{lr}
\hline \multicolumn{2}{l}{$\begin{array}{l}\text { Clínica/hospital tiene comité de ética clínico } \\
\text { Si }\end{array}$} \\
\hline No & $33.0(73.0 \%)$ \\
\hline $\begin{array}{r}\text { La unidad cuenta con una guía de acompañamiento al final de la vida o guía de cuidado } \\
\text { paliativo }\end{array}$ & $12.0 \%)$ \\
\hline Sí & $27.0(40.0 \%)$ \\
\hline No & \\
\hline Influencia de la creencia religiosa en la toma de las decisiones \\
\hline Totalmente de acuerdo & $12.0(26.7 \%)$ \\
\hline De acuerdo & $15.0(33.3 \%)$ \\
\hline Ni de acuerdo/ni en desacuerdo & $12.0(26.7 \%)$ \\
\hline En desacuerdo & $3.0(6.7 \%)$ \\
\hline Totalmente en desacuerdo & $1.0(2.2 \%)$ \\
\hline No tengo creencia religiosa & $2.0(4.4 \%)$ \\
\hline
\end{tabular}

Tabla 3. Procedimientos para resolver el problema ético.

\begin{tabular}{lr}
\hline VARIABLE & $\mathrm{n}(\%)$ / Promedio (DS) \\
\hline Como resuelve el problema ético & \\
\hline Decisión personal del médico tratante & $3.0(20.0 \%)$ \\
\hline Junta médica & $15.0(33.0 \%)$ \\
\hline Consulta a comité de ética médica & $35.0(78.0 \%)$ \\
\hline Concertado en conjunto con los padres y equipo tratante & $0.0(0.0 \%)$ \\
\hline Concertado únicamente con padres & $2.0(4.0 \%)$ \\
\hline Concertado únicamente con equipo tratante & \\
\hline Participa la familia en la toma de la decisión para resolver el problema ético & $37.0(78.0 \%)$ \\
\hline Siempre & $7.0(16.0 \%)$ \\
\hline Casi siempre & $2.0(4.0 \%)$ \\
\hline A veces & $0.0(0.0 \%)$ \\
\hline Casi nunca & $1.0(2.0 \%)$ \\
\hline Nunca & \\
\hline Registra la decisión en la historia clínica & $34.0(76.0 \%)$ \\
\hline Siempre & $4.0(9.0 \%)$ \\
\hline Casi siempre & $3.0(7.0 \%)$ \\
\hline A Veces & $2.0(4.0 \%)$ \\
\hline Casi nunca & $2.0(4.0 \%)$ \\
\hline Nunca & \\
\hline Firman los padres estas decisiones & $29.0(64.0 \%)$ \\
\hline Si & $11.0(24.0 \%)$ \\
\hline A veces & $5.0(11.0 \%)$ \\
\hline No & \\
\hline Se apoya en el comité de ética clínica & $33.0(73.0 \%)$ \\
\hline Si & $12.0(27.0 \%)$ \\
\hline No &
\end{tabular}

El 93.0\% ha limitado el esfuerzo terapéutico (LET); las principales formas de la LET son la no reanimación $82.0 \%$, el no inicio de soporte ventilatorio invasivo $62.0 \%$ y el no inicio del soporte vaso activo $60.0 \%$. El 98.0\% tienen algún límite de viabilidad para no iniciar reanimación en salas de adaptación y el $96.0 \%$ identifican algunas patologías para limitar el esfuerzo terapéutico o no reanimar en salas de adaptación (Tabla 3). Principalmente limitan la reanimación 
Tabla 4. Opinión acerca de ciertos problemas éticos.

\begin{tabular}{|c|c|}
\hline VARIABLE & n (\%) / Promedio (DS) \\
\hline \multicolumn{2}{|l|}{ Alguna vez ha limitado el esfuerzo terapéutico } \\
\hline $\mathrm{Si}$ & $42.0(93.0 \%)$ \\
\hline No & $3.0(7.0 \%)$ \\
\hline \multicolumn{2}{|l|}{ Frecuencia en que ha limitado el esfuerzo terapéutico } \\
\hline Menos de 5 veces & $13.0(31.0 \%)$ \\
\hline Entre 5 y 10 veces & $21.0(50.0 \%)$ \\
\hline Entre 11 y 20 veces & $7.0(16.7 \%)$ \\
\hline Entre 21 y 30 veces & $1.0(2.3 \%)$ \\
\hline \multicolumn{2}{|c|}{ En que basa la decisión de limitar el esfuerzo terapéutico } \\
\hline Tratamiento inútil o fútil & $31.0(69.0 \%)$ \\
\hline Tratamiento desproporcionado & $14.0(31.0 \%)$ \\
\hline Tratamiento no indicado & $5.0(11.0 \%)$ \\
\hline Otro & $5.0(11.0 \%)$ \\
\hline \multicolumn{2}{|l|}{ Considera limitación del esfuerzo terapéutico } \\
\hline No iniciar reanimación & $37.0(82.0 \%)$ \\
\hline Retirar soporte ventilatorio invasivo y no invasivo & $6.0(13.0 \%)$ \\
\hline No iniciar soporte ventilatorio invasivo y no invasivo & $28.0(62.0 \%)$ \\
\hline Retirar soporte vaso activo & $14.0(31.0 \%)$ \\
\hline No iniciar soporte vaso activo & $27.0(60.0 \%)$ \\
\hline Retirar manejo antibiótico & $6.0(13.0 \%)$ \\
\hline No iniciar manejo antibiótico & $14.0(31.0 \%)$ \\
\hline Retirar Nutrición parenteral & $11.0(24.0 \%)$ \\
\hline No iniciar Nutrición parenteral & $15.0(33.0 \%)$ \\
\hline Retirar nutrición enteral & $1.0(2.0 \%)$ \\
\hline No iniciar nutrición enteral & $3.0(7.0 \%)$ \\
\hline Limita ingreso a la unidad neonatal & $7.0(16.0 \%)$ \\
\hline No administra líquidos endovenosos & $1.0(2.0 \%)$ \\
\hline Ninguna de las anteriores & $5.0(11.0 \%)$ \\
\hline
\end{tabular}

Tiene algún límite de viabilidad (peso o edad gestacional) o patología para NO iniciar reanimación al nacimiento

\begin{tabular}{lr}
\hline $\mathrm{Si}$ & $44.0(98.0 \%)$ \\
\hline No & $1.0(2.0 \%)$ \\
\hline Peso al nacer & \\
\hline Promedio & \\
\hline Máximo & $457.0 \mathrm{gr}(\mathrm{DS}+/-86.6 \mathrm{gr}$ \\
\hline Mínimo & \\
\hline Edad gestacional & 24.6 semanas (DS +/- 1.3$)$ \\
\hline Promedio & 27.0 semanas \\
\hline Máximo & 22.0 semanas \\
\hline Mínimo & \\
\hline Patología/cromosomopatía & $35.0(78.0 \%)$ \\
\hline Trisomía 13 & $32.0(71.0 \%)$ \\
\hline Trisomía 18 & $23.0(51.0 \%)$ \\
\hline Anencefalia & $3.0(7.0 \%)$ \\
\hline Malformaciones mayores & $1.0(2.0 \%)$ \\
\hline Holoprosencefalia & $1.0(2.0 \%)$ \\
\hline Ventrí́culo único & $1.0(2.0 \%)$ \\
\hline Agenesia renal bilateral &
\end{tabular}




\begin{tabular}{lr}
\hline \multicolumn{2}{l}{ Ha estado expuesto a situaciones que usted considere encarnizamiento terapéutico } \\
\hline $\mathrm{Si}$ & $39.0(87.0 \%)$ \\
\hline $\mathrm{No}$ & $6.0(13.0 \%)$ \\
\hline Acompañamiento al final de la vida & \\
\hline Holística espiritual & $29.0(64.0 \%)$ \\
\hline Psicosocial (apoyo psiquiatría/psicología) & $39.0(87.0 \%)$ \\
\hline Intervenciones para prevenir/aliviar sufrimiento al recién & $39.0(87.0 \%)$ \\
nacido & 0.0 \\
\hline Ninguna & $1.0(2.2 \%)$ \\
\hline Otros & \\
\hline
\end{tabular}

en salas de adaptación por el peso al nacer, con un promedio de menos de 557.0 gr para tomar la decisión de no reanimar y entre los que tienen en cuenta la edad gestacional en promedio la toman de 24.6 semanas. En cuanto a patologías asociadas para no reanimar en salas de adaptación están las cromosopatías como primera causa y, entre estas, la trisomía 13 y trisomía 18. Ver (Tabla 4).

\section{Discusión}

El presente estudio caracteriza la forma en que los profesionales encargados del cuidado del neonato, se enfrentan y opinan ante ciertos problemas éticos. La mayoría eran neonatólogos, con experiencia en el cuidado crítico del neonato de alta complejidad; todos se han enfrentado a dilemas éticos.

Integrar el cuidado paliativo en la unidad puede ser difícil principalmente por la formación insuficiente del personal (17). Estar capacitados en bioética ayuda en la resolución del problema, y en los programas de neonatología aporta el conocimiento para entender y discutir temas de decisiones éticas difíciles $(18,19)$. Otros autores muestran que los especialistas en cuidado intensivo adultos, pediátrica y neonatal, manifiestan la necesidad de capacitarse en temas éticos, el $97.6 \%$ consideraban que se debía incluir en el programa académico de la especialidad; sólo el $40.0 \%$ habían recibido capacitación. En nuestro estudio, el 60.0\% de los profesionales reconocían haber tenido capacitación en temas éticos (20).

La Academia Americana de Pediatría, ha promovido los comités de ética clínica, recomendando disponibilidad en instituciones con unidades neonatales para apoyo al profesional y padres (21). En este estudio, la mayoría de profesionales cuenta con comité de ética en sus sitios de práctica, pero es llamativo el uso infrecuente de estos, ya que sólo el $33.0 \%$ consultan cuando se enfrentan a dilemas éticos. Autores como Rincón et al, encontraron datos similares donde el $64.0 \%$ cuentan con comité de ética, pero solicitan su apoyo siempre en el $22.0 \%$ (20). Se recomienda, en la literatura, que para la toma de estas decisiones se involucre a todo el equipo tratante, padres y otros profesionales que puedan dar luz en la decisión y en todo caso limitar los casos en los que se tome solo (22). En nuestro estudio, al resolver el problema ético, el $67.0 \%$ opta por junta médica y el $78.0 \%$ concertado con los padres y equipo tratante; pocos se apoyan en comités de ética (33.0\%) y nadie lo concierta únicamente con los padres; como dato interesante, la frecuencia con que se involucra a los padres (siempre 78.0\%), se registra la decisión en la historia clínica (siempre 76.0\%) y se firma por parte de los padres (siempre 64.0\%) la decisión es alta; esto concuerda con lo recomendado en la literatura, en donde se recomienda que las decisiones queden consignadas y firmadas en la historia clínica (17). Por otra parte, entre médicos de UCI pediátrica de España, en un estudio para caracterizar las decisiones difíciles en las unidades neonatales, encontraron que la participación de familiares es muy variable y no siempre queda consignada en la historia clínica (5). Se recomienda que el médico en la unidad neonatal tome la decisión apoyado en sus pares, equipo tratante, médicos de mayor experiencia y en el comité de ética médica $(14,17)$. Sin embargo, en cuanto involucrar a los padres en la decisión, la opinión en la literatura de los neonatólogos es variable y sin consenso (18).

La principal forma de limitar el esfuerzo terapéutico (LET) en neonatos es la decisión de no iniciar soporte vital y/o con orden de no reanimar $(23,24)$. En el estudio, el $97.0 \%$ han LET, y la mayoría en más de una ocasión, limitan con orden de no reanimación en salas de adaptación y limitando el soporte vital, principalmente no iniciando ventilación mecánica o soporte vaso activo; menos frecuente retirando tratamientos vitales iniciados como la ventilación mecánica. En estudios similares para caracterizar las decisiones difíciles entre profesionales de unidades neonatales de alta complejidad se ha encontrado que se realiza LET principalmente con el retiro del soporte vital, dentro de los cuales se destaca la ventilación mecánica y no iniciando reanimación neonatal $(22,25)$. En estos mismos estudios, al caracterizar los criterios utilizados por los profesionales en este tipo de decisiones, se observa que tienen en cuenta el pronóstico del paciente desde el punto de vista de la probabilidad de supervivencia a corto plazo y el juicio de la calidad de vida $(17,25,26)$. En este estudio la toma de la decisión se basó principalmente en que el tratamiento fuera inútil, fútil o desproporcionado. 
En el estudio, la mayoría tienen presentes límites de viabilidad para no iniciar reanimación en salas de adaptación, principalmente el peso al nacer y con menos frecuencia la edad gestacional; para el peso, el límite es principalmente 500.0 gr y 25.0 semanas para la edad gestacional (EG). Tienen presente algún tipo de malformación congénita para no iniciar reanimación, las más frecuentes son Trisomía 13, Trisomía 18 y anencefalia. En otro estudio se encontró que entre profesionales de unidades neonatales que las causas más frecuentes para no reanimar fueron las malformaciones congénitas $(47.0 \%)$, la asfixia perinatal y la hemorragia intracraneal (37.0\%) (25). Por otra parte, en Estados Unidos, los profesionales encargados de la atención del recién nacido en salas de parto, casi nunca inician reanimación neonatal en menores de 23.0 semanas y/o de 500.0 gr mientras que el $90.0 \%$ iniciaría reanimación en mayores de 25.0 semanas y/o 600.0 gr de peso al nacer; para los recién nacidos entre las 23.0 y 24.0 semanas de edad gestacional y con pesos entre 500.0 y 600.0 gramos centran la decisión con la opinión de los padres y el estado del bebé al nacer (27).

Es interesante analizar la EG como criterio de decisión. Andrews y et al, realizaron un estudio con el objetivo de evaluar si la edad gestacional era un factor que marcara el neurodesarrollo a los 2 años de vida, en neonatos entre 23.0 - 28.0 semanas de edad gestacional (28). Encontrando que, entre los sobrevivientes de los diferentes grupos, no hubo diferencias en el neurodesarrollo, y no mejoró el desenlace entre los de mayor EG (28). Se ha planteado que la decisión de reanimar con base en la EG pueda ser una clase de discriminación, en donde se le pueden negar tratamientos con base en un solo dato aleatorio y no exacto, sin tener en cuenta otras variables biológicas que influencian el desenlace y pronóstico (29); por lo que se plantean alternativas como decisiones individualizadas, el pronóstico y decisión compartida con los padres junto con la ponderación de la edad gestacional (29).

La influencia de variables biológicas es importante en la decisión del especialista para limitar o continuar con el tratamiento. En un estudio de cohorte para estudiar factores de riesgo de alteración en el neurodesarrollo en bebés entre 22.0 a 25.0 semanas de EG, encontraron que la EG sola no marca pronóstico, sino que hay otros factores como el sexo, el uso de corticoide antenatal y el peso al nacer que se deben tener en cuenta y en cuanto al peso, por cada $100.0 \mathrm{gr}$ de aumento en el peso al nacer disminuye el riesgo de muerte y alteración en el neurodesarrollo(30).

En cuanto a la discusión de la EG como criterio para no iniciar la reanimación neonatal, el comité del feto y el Recién Nacido de la Sociedad de Pediatría de Canadá (31), recomienda no utilizarla, ya que esta puede ser inexacta en su cálculo y no asociarse al desenlace, puede ser utilizada en conjunto con otros indicadores de pronóstico; enfatizando siempre la comunicación con los padres y realizando una consulta antenatal, compartiendo información de sobrevida y desenlaces a corto y largo plazo y evidencia de calidad de vida(31).
Estos datos son interesantes para las unidades de nuestro país ya que observamos entre los profesionales encuestados una frecuencia alta de decisiones éticas difíciles en su práctica clínica, con situaciones complejas para resolver; frecuentemente limitan el esfuerzo terapéutico y tienen límites de viabilidad tanto para el peso y la edad gestacional así como patologías que les hacen tomar la decisión de no reanimar o el limitar el esfuerzo terapéutico, pero contrasta con el poco apoyo en el comité de ética clínica para la decisión.

Datos importantes para generar la discusión de si es posible la unificación de conceptos y criterios entre las unidades con miras a generar protocolos y guías al respecto. Adicionalmente, el dato en el que la familia es involucrada en la decisión junto con los pares de la unidad es muy interesante para tener en cuenta y animar a que se continúe en este camino. Igualmente, los datos de caracterización del estudio son importantes para poder recomendar a las personas encargadas de la dirección de las unidades que generen recomendaciones para que sus profesionales especialistas se capaciten en estos temas para una mejor práctica clínica y abordaje de estas situaciones.

Como limitación del presente estudio se puede plantear el número de profesionales que contestaron la encuesta, ya que se esperaba que podría ser un número mayor para tener una muestra más representativa; sin embargo, anotamos que los que respondieron son de diferentes unidades lo que puede darnos una muestra de la caracterización en estas.

\section{Conclusiones}

El 100\% del personal especialista en las unidades neonatales, se ha enfrentado a problemas éticos y la gran mayoría en más de una vez, lo que muestra la importancia y actualidad del tema dentro de la práctica clínica. Ven importante la capacitación académica en estos temas y ante el mismo problema ético la forma de actuar entre especialistas puede ser diferente. Se percibe una falta de guías para enfrentar estos dilemas en la práctica. Es interesante que al enfrentar y tomar las decisiones involucran de manera frecuente a los padres y la decisión la registran en la historia clínica. En su práctica clínica tienen presentes límites de viabilidad para la decisión de no reanimación, principalmente el peso al nacer y patologías asociadas al nacimiento. La forma más frecuente de limitar el esfuerzo terapéutico es la decisión de no iniciar soporte vital (ventilación mecánica o soporte vaso activo) y/o la orden de no reanimación en la sala de adaptación.

\section{Conflicto de intereses}

Los autores declaran libremente no tener conflicto de intereses. 


\section{Referencias}

1. Messner H, Gentili L. Reconciling ethical and legal aspects in neonatal intensive care. J Matern Fetal Neonatal Med. 2011; 24(S1): 126-8.

2. Rellan Rodríguez S, García de Ribera C, Aragón García M. El recién nacido prematuro. AEP-SEN (Protocolos Diagnóstico Terapeútico de la AEP: Neonatología). Ed 2, España 2008; 68-78.

3. Hübner ME, Ramírez R. Sobrevida, viabilidad y pronóstico del prematuro. Rev Médica Chile. 2002; 130(8): 931-8.

4. Guellec I, Lapillonne A, Renolleau S, Charlaluk M-L, Roze J-C, Marret S, et al. Neurologic Outcomes at School Age in Very Preterm Infants Born With Severe or Mild Growth Restriction. Pediatrics. 2011; 127(4): e883-91.

5. Hernández-González A, Tezanos H, HernándezRastrollo R, Cambra-Lasaosa F, Rodríguez Núñez A, Failde I. Encuesta de ética en las unidades de cuidados intensivos pediátricos españolas. Anales de Pediatría 2006; 64(6): 542-9.

6. Arce Casas A, Iriondo Sanz M, Krauel Vidal J, Jiménez González R, Campistol Plana J, Poo Argüelles P, et al. Seguimiento neurológico de recién nacidos menores de 1.500 gramos a los dos años de edad. Anales de Pediatría 2003; 59(5): 454-61.

7. Garduño Espinosa A, Muñoz Ramírez R, Olivares Díaz C. Dilemas éticos y toma de decisiones en Unidades de Cuidados Intensivos Neonatales. Bol Méd Hosp Infant México. 2010; 67(3): 259-69.

8. Sebastiani M, Ceriani J. Aspectos bioéticos en el cuidado de los recién nacidos extremadamente prematuros. Arch Argent Pediatr. 2008; 106(3): 242-8.

9. Torres JT, García LA. Toma de decisiones éticas y limitación del soporte vital en recién nacidos críticos. Esp Pediatr. 1997; 46: 53-9.

10. Bórquez G, Anguita V, Bernier L. El prematuro en cuidado intensivo neonatal ¿Cuándo es el momento de decir no más? Reflexión bioética en torno a la limitación del esfuerzo terapéutico. Rev Chil Pediatría. 2004; 75(2): $181-7$.

11. Jiménez R, Molina $V$. Bases éticas en Neonatología Decisiones de tratamiento selectivo en recién nacidos Normas básicas de actuación Protocolos Diagnóstico Terapeúticos de la AEP. Neonatología. 2008; 54: 519-21.

12. Hübner ME, Ramírez R. Sobrevida, viabilidad y pronóstico del prematuro. Rev Médica Chile. 2002; 130(8): 931-8.

13. Thompson J. Los principios de ética biomédica. Rev Pediatr Colomb. 2005; (4): 15-34.

14. Kuschel CA, Kent A. Improved neonatal survival and outcomes at borderline viability brings increasing ethical dilemmas. J Paediatr Child Health. 2011; 47(9): 585-9.

15. Burns JP, Mitchell C, Griffith JL, Truog RD. End-of-life care in the pediatric intensive care unit: attitudes and practices of pediatric critical care physicians and nurses. Crit Care Med. 2001; 29(3): 658-64.

16. Randolph AG, Zollo MB, Wigton RS, Yeh TS. Factors explaining variability among caregivers in the intent to restrict life-support interventions in a pediatric intensive care unit. Crit Care Med. 1997; 25(3): 435-9.
17. Torres JT, de Heredia Goya JL, Rubia NH, Jimenez PN, Munóz FG, Rodríguez JP. Recomendaciones sobre toma de decisiones y cuidados al final de la vida en neonatología. anales de pediatría 2013; 78(3): 190.e1e14.

18. Swinton $\mathrm{CH}$, Lantos JD. Current empirical research in neonatal bioethics. Acta Paediatr 2010; 99(12): 1773-81.

19. Alonso M, Alonso M, López R. Dilemas éticos de las decisiones médicas en cuidados intensivos. Acta Médica del Centro. 2014; 8(1): e1-e9.

20. Rincón M, Carrillo Becerra LM, Camargo L, Amparo M, Guerrero Guzmán M del P, Gutiérrez Cardona NA, et al Educación, bioética y toma de decisiones éticas en unidades de cuidado intensivo. Rev Latinoam Bioét. 2008; 8(2): 114-23.

21. Mercurio MR. The role of a pediatric ethics committee in the newborn intensive care unit. J Perinatol. 2011 Jan; 31(1): 1-9.

22. Torres JT, García LA. Toma de decisiones éticas y limitación del soporte vital en recién nacidos críticos. Esp Pediatr. 1997; 46: 53-9.

23. da Costa DE, Ghazal H, Al Khusaiby S. Do Not Resuscitate orders and ethical decisions in a neonatal intensive care unit in a Muslim community. Arch Dis Child Fetal Neonatal Ed. 2002; 86(2): F115-9.

24. González AH, Rastrollo RH, Lasaosa FC, Núñez AR, Failde I, de la SECIP a de É. Encuesta de ética en las unidades de cuidados intensivos españolas. An Pediatr 2006; 64 (6):542-9.

25. Tejedor Torres JC. Decisiones de limitación del esfuerzo terapéutico en recién nacidos críticos: estudio multicéntrico. An Pediatría. 2002;57(6): 547-53.

26. Torres JT, García LA. Toma de decisiones éticas y limitación del soporte vital en recién nacidos críticos. Esp Pediatr. 1997; 46: 53-9.

27. Singh J, Fanaroff J, Andrews B, Caldarelli L, Lagatta J, Plesha-Troyke S, et al. Resuscitation in the 'gray zone' of viability: determining physician preferences and predicting infant outcomes. Pediatrics. 2007; 120(3): 519-26.

28. Andrews B, Lagatta J, Chu A, Plesha-Troyke S, Schreiber M, Lantos J, et al. The nonimpact of gestational age on neurodevelopmental outcome for ventilated survivors born at 23-28 weeks of gestation. Acta Paediatr. 2012; 101(6): 574-8.

29. Wilkinson DJ. Gestational ageism. Arch Pediatr Adolesc Med. 2012; 166(6): 567-72.

30. Tyson JE, Parikh NA, Langer J, Green C, Higgins RD. Intensive care for extreme prematurity-moving beyond gestational age. N Engl J Med. 2008; 358 (16): 1672-81.

31. Janvier A, Barrington KJ, Aziz K, Bancalari E, Batton D, Bellieni $\mathrm{C}$, et al. CPS position statement for prenatal counselling before a premature birth: simple rules for complicated decisions. Paediatr Child Health. 2014; 19 (1): 22. 